PAPER • OPEN ACCESS

\section{A method for the measurement of mass and number of graphene oxide sheets in suspension based on non-spherical approximations}

To cite this article: Livia Elena Crica et al 2021 2D Mater. 8035044

View the article online for updates and enhancements.
You may also like

\begin{tabular}{l} 
Development of a ridged microstrip \\
$\frac{\text { microwave interferometer for plasma }}{\text { electron density measurements }}$ \\
\hline C H Hsieh, Y W Liang, J Y Jeng et al. \\
- Optical Coherence Tomography \\
$\frac{\text { Velocimetry with Complex Fluids }}{\text { A Malm, T A Waigh, S Jaradat et al. }}$ \\
$-\frac{\text { Magnetic heating characteristics of }}{\text { La }{ }_{\text {Sr. }} \mathrm{Ca}_{0 .} \mathrm{MnO}_{3} \text { nanoparticles }}$ \\
$\frac{\text { fabricated by a high energy mechanical }}{\text { milling method }}$ \\
Hung Manh Do, Hong Nam Pham, Van \\
Chien Nguyen et al.
\end{tabular}


CrossMark

OPEN ACCESS

RECEIVED

11 January 2021

REVISED

26 April 2021

ACCEPTED FOR PUBLICATION

4 May 2021

PUBLISHED

2 June 2021

Original content from

this work may be used

under the terms of the

Creative Commons

Attribution 4.0 licence.

Any further distribution of this work must

maintain attribution to

the author(s) and the title

of the work, journal

citation and DOI.

PAPER

\title{
A method for the measurement of mass and number of graphene oxide sheets in suspension based on non-spherical
} approximations

\author{
Livia Elena Crica ${ }^{1}\left(\mathbb{0}\right.$, Thomas James Dennison ${ }^{2}$, Elise Andrea Guerini ${ }^{2}$ and Kostas Kostarelos ${ }^{1,3, *}(\mathbb{C}$ \\ 1 Nanomedicine Lab, National Graphene Institute and Faculty of Biology, Medicine \& Health, The University of Manchester, \\ Manchester M13 9PT, United Kingdom \\ 2 Malvern Panalytical Ltd, Grovewood Road, Malvern, Worcestershire WR14 1XZ, United Kingdom \\ 3 Catalan Institute of Nanoscience and Nanotechnology (ICN2), UAB Campus Bellaterra, Barcelona, Spain \\ * Author to whom any correspondence should be addressed. \\ E-mail: kostas.kostarelos@manchester.ac.uk
}

Keywords: RMM, buoyant mass, particle, 2D materials

Supplementary material for this article is available online

\begin{abstract}
Currently, particle analysis of 2D materials in suspension is commonly restricted to microscopic techniques in the dry state, and thus does not permit an accurate investigation of colloidal suspensions. Colloids in bulk can be assessed by light scattering and diffraction to investigate features such as their hydrodynamic size, charge and concentration. However, the main drawback of such techniques lies in the application of analytical and computational methods based on models assuming particle sphericity which are not representative for 2D materials. Resonance mass measurement (RMM) is a technique which can enable the analysis of 2D materials in suspension without the assumptions of spherical models. Here, we report the application of RMM to measure particle mass and concentration for three types of graphene oxide (GO) aqueous dispersions. Using micro- and nano-suspended resonating sensors, we were able to decipher gravimetric differences between GO and graphitic materials. Our results support the urge for proper definitions and standardisations of graphene based materials, and offer a new method of characterisation for 2D material colloids in liquid suspension.
\end{abstract}

\section{Introduction}

Two-dimensional (2D) materials, starting with graphene-based materials (GBMs), are expected to be incorporated into a vast range of applications from electronics to materials science and from aerospace engineering to biomedicine (Novoselov et al 2012, Dimitrios et al 2013, Randviir et al 2014). However GBMs come in various forms, with different properties: different lateral size and thickness distribution, oxidation degree and purity, often leading to contradictions within the scientific community (Bianco 2013, Wick et al 2014, Kauling et al 2018). There is an urgent need for proper definitions and standardisation for GBMs which are often described with similar product names despite several and significant deviations in physicochemical characteristics, thereby presenting an obstacle to the industrial adoption and translation of these innovative materials (Bianco et al
2013, Bøggild 2018). For this reason, good practice guidelines and standards are under development by various institutions (Bianco et al 2013, ISO 2017, Pollard and Clifford 2017, Kauling et al 2018, Pollard 2017).

Currently, microscopic techniques, such as optical microscopy (OM), scanning and transmission electron microscopy (SEM and TEM) and atomic force microscopy (AFM) are the most commonly used methodologies for the analysis of individual GBM sheets (Pollard 2017). However, when 2D sheets are in colloidal suspension, microscopic analysis of GBMs in a dry state is not ideal. This is because sheets can fold, wrinkle, bundle or stack to adopt different conformations, either when freely floating in different fluids or during the drying processes applied, thereby affecting various properties, such as their size, thickness and density or even mass (Whitby et al 2012). Common methods to analyse particles 

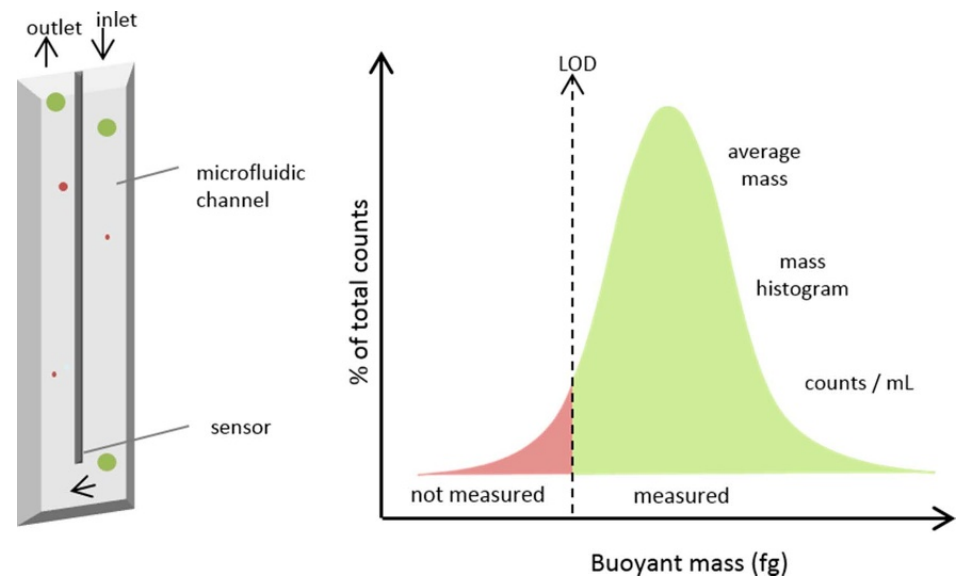

Figure 1. Illustration of the RMM principle: particles flow through the microfluidic channel and change the resonant frequency of the sensor. The frequency shift caused by each particle is translated to buoyant mass. As particles are measured individually in a known volume, the number of counts per millilitre is approximated. The limit of detection (LOD) separates the background noise and undetectable particles from the particle count.

in suspension are dynamic light scattering (DLS) and nanoparticle tracking analysis (NTA), which explore the light scattered by particles in Brownian motion to approximate their size. However, both techniques rely on spherical models which are unsuitable for flat and irregularly shaped nanomaterials such as GBMs.

An alternative technique for the analysis of suspended particles is resonance mass measurement (RMM). RMM is an innovative and emerging technique used to characterise and count particles within the subvisible and submicron range (Weinbuch et al 2013, Höldrich et al 2017). At the heart of the technology lies a microelectromechanical sensor comprising a resonating cantilever with a microfluidic channel, through which suspended particles are measured under flow (figure 1). When a particle between $50 \mathrm{~nm}$ and $5 \mu \mathrm{m}$ flows through the cantilever it will cause a shift in the cantilever's resonant frequency, should the density of the particle differ to the fluid in which it is dispersed. This frequency shift indicates the buoyant mass of the particle being measured; denser materials will cause a negative frequency shift and have a positive buoyant mass, whereas less dense materials will cause a positive frequency shift and have a negative buoyant mass. This can be exploited to discriminate between positively and negatively buoyant particles within a sample. The extent of the frequency shift directly relates to the particle's buoyant mass, from which the dry mass can be inferred as well as particle volume and diameter (assuming a spherical shape). Since particles flow through the cantilever individually and are counted in a known volume, RMM can accurately determine particle concentration. Furthermore, given the ability to measure individual particles and the extreme sensitivity of this technique (RMM is able to measure particle mass as low as $10^{-18} \mathrm{~g}$ ), highresolution particle mass distribution information is inherently obtained. We foresee that characterising
GBMs by mass in this way could be more valuable than reporting a spherical size by light scattering techniques, since these materials are two-dimensional.

So far, RMM has proved useful in the analysis of mammalian and bacterial cells, in identifying protein aggregates, and characterising nanomaterials and nanobubbles (Nittayacharn et al 2018, Krueger et al 2019, Pollo et al 2019). Son et al measured the change in volume and density of cells undergoing mitosis to discover that cells swell and reduce their density as a result of intracellular water regulation (Son et al 2015). Lewis et al used RMM to compare the live and dead bacterial populations and monitor bacterial growth using real time monitoring of cell concentration (2014). The change in mass of nanomaterials upon surface modification was also assessed by RMM. Höldrich et al used this technique to determine the number of pepsin molecules adsorbed on gold nanoparticles, the results of which were in agreement with commercial protein quantification kits (2017). Similarly, Nejadnik et al studied the interaction of bovine serum albumin and monoclonal human antibody with polystyrene nanoparticles and showed a shift in particle mass which could not be determined with other techniques (Nejadnik and Jiskoot 2015). RMM can also be used to differentiate particles based on their buoyancy where size-dependent techniques fail. In pharmaceutical sciences for instance, RMM can be used to detect positively buoyant contaminants such as silicon oil in drug formulations that contain negatively buoyant particles (e.g. protein aggregates) (Weinbuch et al 2013, Pollo et al 2019). In a similar way, ultrafine bubbles can be distinguished from other types of particles in suspensions by measuring their positively buoyant mass (Kobayashi et al 2014).

However, due to its relative novelty, RMM is currently an insufficiently explored technique which could serve sectors where the particle number and 
mass in suspension is of interest but difficult to obtain with conventional techniques. For the characterisation of GBMs in aqueous dispersions in particular, we theorised that RMM could be more precise in measuring the particle mass and concentration of GBMs than other more traditional techniques. Indeed, the uniqueness of this technique stands in its ability to measure individual particles and clusters/aggregates in fluids without using spherical models, a major drawback of many alternative techniques for $2 \mathrm{D}$ materials. Herein, we characterised the physicochemical properties, including the particle mass and concentration by RMM, of three types of graphene oxide (GO) aqueous dispersions, including one prepared in-house and two commercial products. To the best of our knowledge, these are the first mass measurements of GO sheets in an aqueous dispersion. We report the added value of measuring buoyant mass using RMM as an extremely sensitive technique that complements conventional microscopy. This new characterisation parameter could be of interest in the undergoing development of standards for the production and quality control of available or emerging $2 \mathrm{D}$ materials, where the same product name still serves as an umbrella for several materials despite their different physicochemical properties.

\section{Results and discussion}

To briefly check the origin of the three materials, we evaluated their physicochemical, structural and morphological properties. Lab GO and Commercial GO-A presented the typical Raman fingerprints, whereas the spectra of Commercial GO-B indicated the existence of graphitic domains by the splitting of the $G$ band to $G+D^{\prime}$ and by the presence of a sharp 2D peak (figure 2(A)) (Pimenta et al 2007). This is also reflected by the reduced metric of disorder $\left(I_{\mathrm{D}} / I_{\mathrm{G}}\right.$ ratio) found for the Commercial GO-B $\left(I_{\mathrm{D}} / I_{\mathrm{G}}=1.00 \pm 0.01\right)$ in comparison to the other two samples ( $\mathrm{Lab} \mathrm{GO}=1.23 \pm 0.03$; Commercial GO-A $=1.42 \pm 0.01)$. Furthermore, the UV-VIS absorbance spectra of Commercial GOB (figure 2(B)) suggests a more graphitic structure, free from functional groups, rather than a GO-specific one (Alam et al 2018). These indications correlate with our observations of the poor colloidal stability of Commercial GO-B in water (figure S1 (available online at stacks.iop.org/2DM/8/ 035044/mmedia)). In contrast, the absorbance bands at $230 \mathrm{~nm}\left(\pi \rightarrow \pi^{*}\right.$ transition of $\mathrm{C}=\mathrm{C}$ bonds $)$ and $310 \mathrm{~nm}\left(n \rightarrow \pi^{*}\right.$ electron transitions in the $\mathrm{C}=\mathrm{O}$ groups) shape the fingerprint of highly exfoliated and oxidised GO (Paredes et al 2008, Dhifaf et al 2016), for both Lab GO and Commercial GO-A samples. As a consequence of the oxidised nature, which inhibits platelet re-stacking via electrostatic repulsions (Dreyer et al 2010), both Lab GO and Commercial GO are stable colloids in MilliQ water (figure S1).
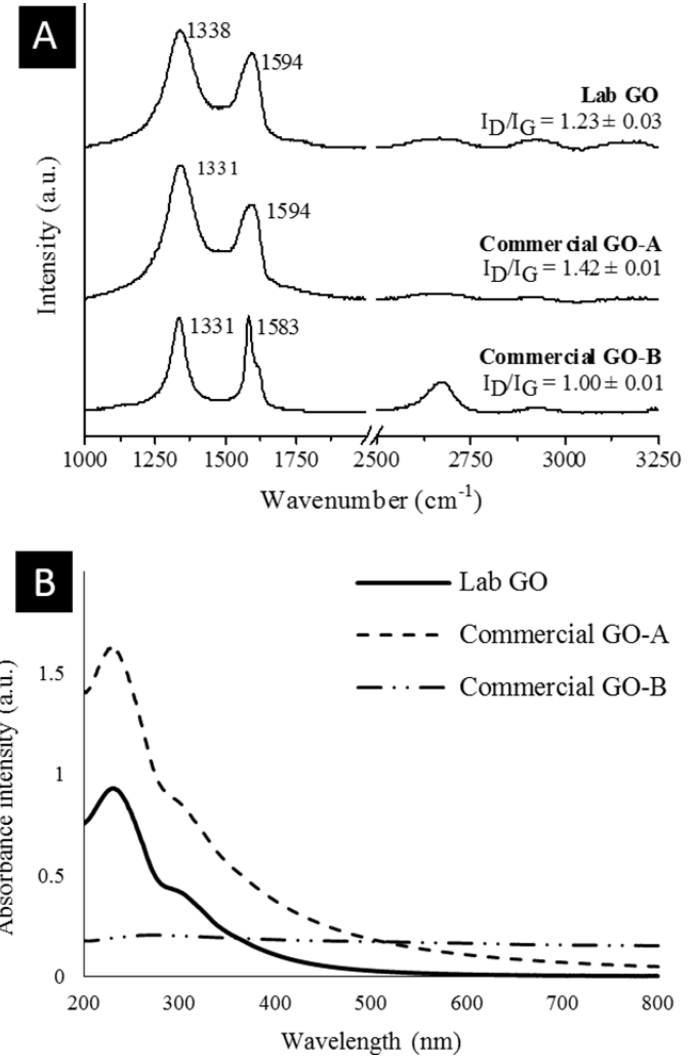

Figure 2. GO material spectroscopic characterisation: structural differences between the lab prepared and commercial GO suspensions, as determined by (A) Raman spectroscopy and (B) UV-VIS spectroscopy. $I_{\mathrm{D}} / I_{\mathrm{G}}$ values represent the mean value $\pm \mathrm{SD}$ of three measurements per sample.

Colloidal stability is of great importance for RMM measurements: at high sedimentation rates, a fraction of the sheet population might be inaccurately measured, leading to poor measurements.

Microscopy analysis using TEM and AFM showed predominantly small and thin layers within the Lab GO (figure 3(A)) and larger thin sheets in Commercial GO-A (figure 3(D)), whereas Commercial GO-B contained mostly large and thick agglomerates (figure 3(G)). Overall, Lab GO contained monolayers with lateral sizes up to $1 \mu \mathrm{m}$ (figures $3(\mathrm{~B})$ and (C)). Single-layers also dominated in the Commercial GO-A sample, however their lateral size spanned from $\sim 0.5$ to a few tens of $\mu \mathrm{m}$ (figures $3(\mathrm{E})$ and $(\mathrm{F})$ ), while Commercial GO-B contained mainly aggregates with $>100 \mathrm{~nm}$ thickness and micrometric sizes (figures $3(\mathrm{H})$ and $(\mathrm{I})$ ).

To assess the mass via RMM and because the lateral size of the two commercial GO samples exceeded $1 \mu \mathrm{m}$ (size limit for the nanosensor), we opted to use only the microsensor, while the Lab GO was analysed using both sensors. Figure 4(A) shows the buoyant mass distribution of the three types of GO measured with the micro-sensor.

Despite all materials having their maximum located at a similar buoyant mass, the percentage of the 

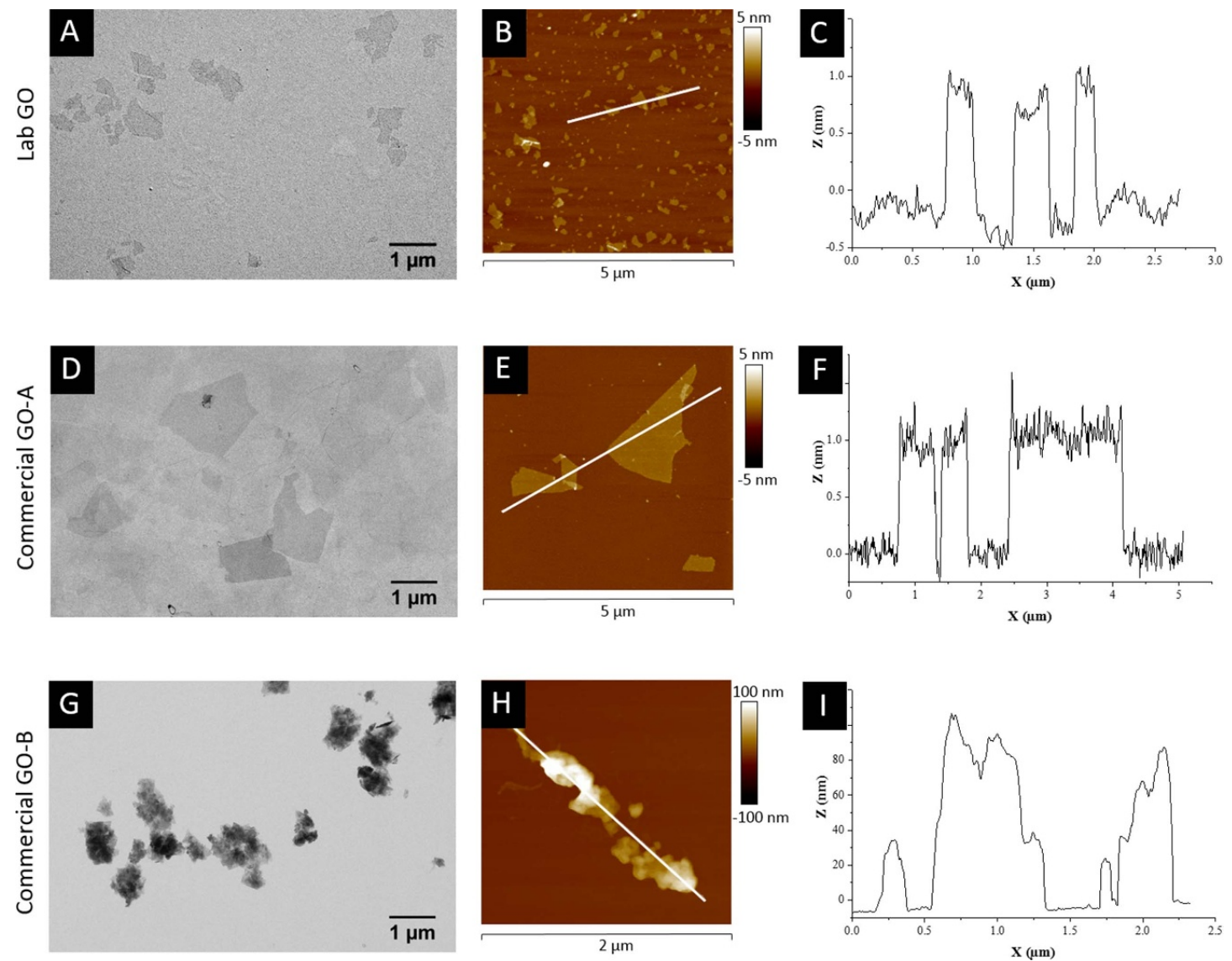

Figure 3. GO material morphology: aspect, lateral size and thickness determined by TEM and AFM for (A)-(C) Lab GO, (D)-(F) Commercial GO-A and (G)-(I) Commercial GO-B. Figures (C), (F) and (I) show the size and thickness of the flakes sectioned within the AFM images (B), (E) and (H), respectively.

total counts was higher for the Lab GO. Moreover, $\mathrm{Lab}$ GO spanned from $6.18 \mathrm{fg}$ (LOD micro) to $84 \mathrm{fg}$, while the commercial GO-A and -B contained particles with buoyant mass up to 968 and $12400 \mathrm{fg}$, respectively. The narrower mass distribution of $\mathrm{Lab}$ GO suggested a lower sample polydispersity. When measured with the nanosensor, the mass distribution of Lab GO ranged from 0.75 to $12.3 \mathrm{fg}$ (figure 4(B)). In these gravimetric ranges, the average buoyant mass of the particles was 11.2 and 1.2 fg for Lab GO (microsensor vs nanosensor), $24 \mathrm{fg}$ for Commercial GO-A and $28 \mathrm{fg}$ for Commercial GO-B (figure 4(C)).

Next, the particle number $n(\# / \mathrm{mg})$ reported in figure 4(D) was calculated using the following formula:

$$
n=\frac{C_{\mathrm{p}}\left(\frac{\#}{\mathrm{ml}}\right)}{C_{\mathrm{m}}\left(\mathrm{mg} \mathrm{ml}^{-1}\right)}
$$

where $C_{\mathrm{p}}(\# / \mathrm{ml})$ is the particle concentration and $C_{\mathrm{m}}$ $\left(\mathrm{mg} \mathrm{ml}^{-1}\right)$ is the mass concentration of the GO stock. By using this formula instead of simply dividing $1 \mathrm{mg}$ by the average dry mass, we avoid an overestimation, i.e. assuming that all particles in $1 \mathrm{mg}$ of GO would be detectable by the instrument (figure S3).

Based upon previous work (Dhifaf et al 2016, Artur Filipe et al 2018), we expected that Lab GO contained thin particles with a lateral size down to a few tens of $\mathrm{nm}(20-50 \mathrm{~nm}$ by AFM). A fraction of these sheets would lie below the detection limit of both the micro- and nano-sensors of the RMM instrument. In agreement with this, the low particle count of Lab GO measured using the microsensor suggested that the highest fraction of sheets was indeed below the detection limit. This was then confirmed using the nanosensor, which is more sensitive to lighter sheets. As expected, when using this sensor, Lab GO contained a higher number of particles in $1 \mathrm{mg}$ compared to Commercial GO-A and GO-B. All RMM results correlated with the AFM and TEM data, where Lab GO contained thin small sheets, Commercial GO-A had thin large sheets and Commercial GO$\mathrm{B}$ contained larger and thicker graphitic aggregates.

Based on these results, the authors disagree with defining the Commercial B sample as 'graphene oxide' and would highlight the importance in consulting the good practice guides and ISO standard referenced here before labelling such materials (Pollard and Clifford 2017, Pollard 2017, ISO 2017). We believe that the average mass per sheet and total number of sheets in dispersion are complementary information that can be used for a more comprehensive characterisation of GBMs. RMM could also serve as a quality control technique in the production 
A
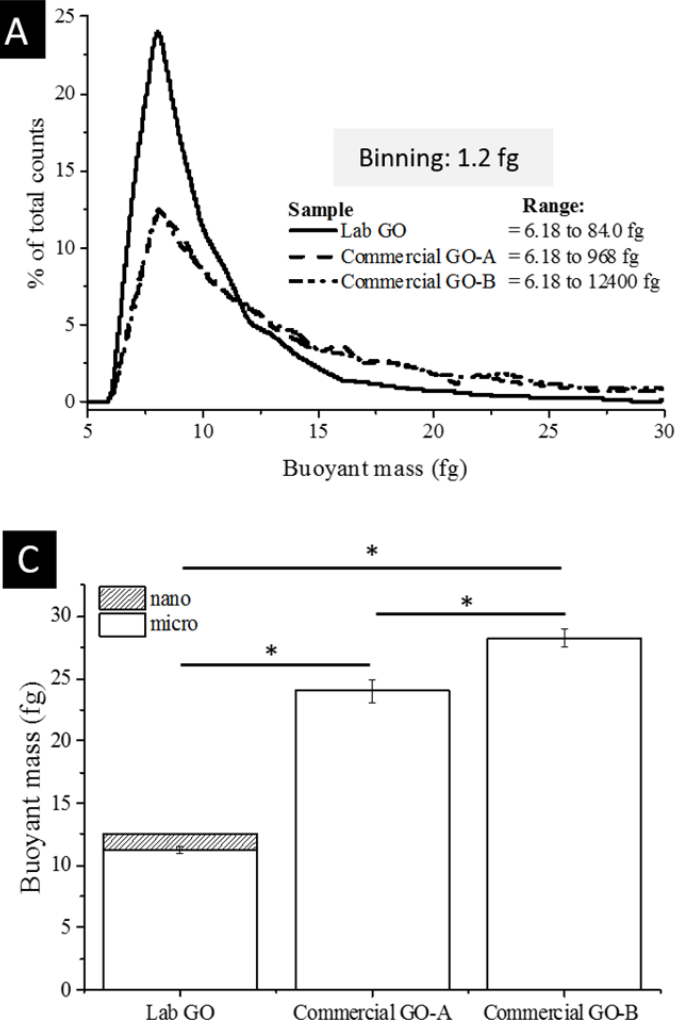

B
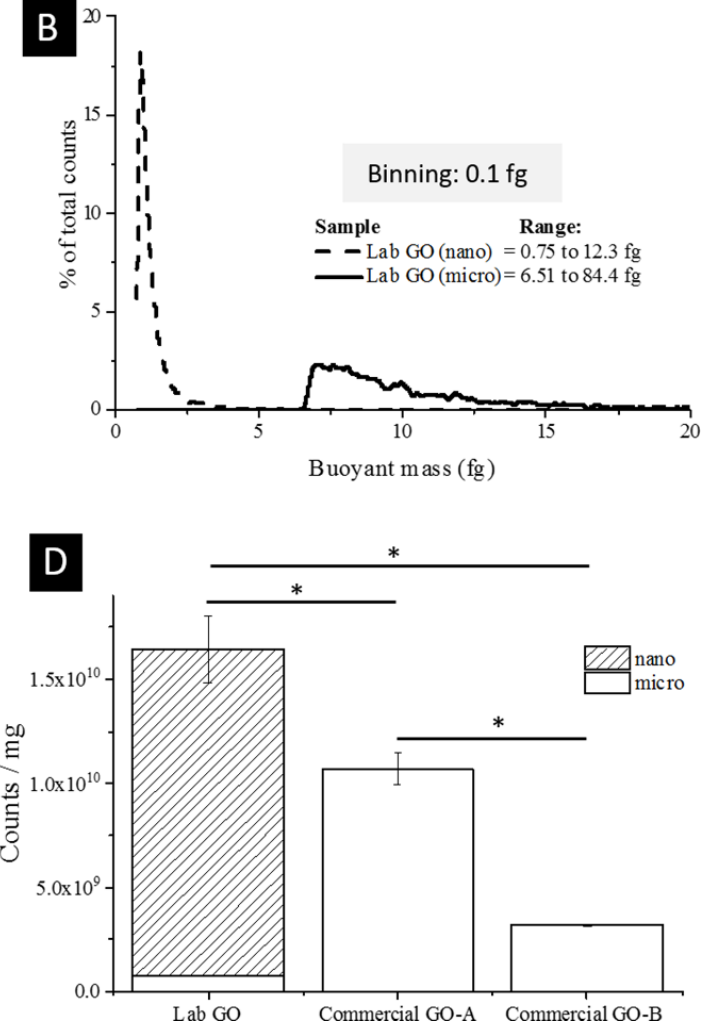

Figure 4. RMM analysis: (A) and (B) buoyant mass distribution of the three types of GO measured with the microsensor and nanosensor, respectively. The nanosensor provides better resolution for measuring the Lab GO as compared with the microsensor. $(\mathrm{C})$ and $(\mathrm{D})$ Average buoyant mass $( \pm \mathrm{SD})$ and counts/mg $( \pm \mathrm{SD})$ of Lab GO compared with Commercial GO-A and -B. Each sample was measured in triplicate, with 1500 counts per replicate. Statistical significance was assessed using one-way ANOVA, with ${ }^{*}<0.01$.

A

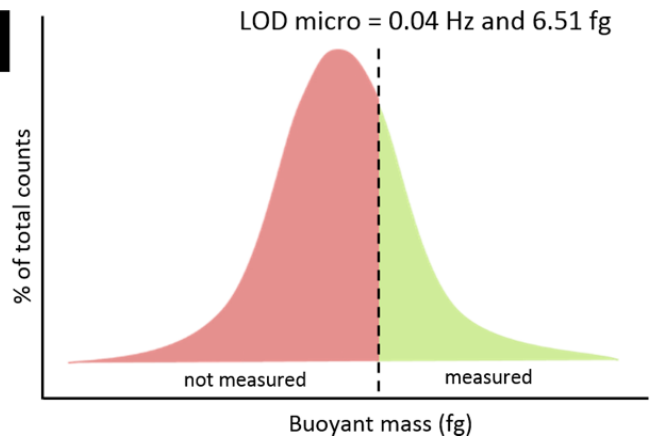

B

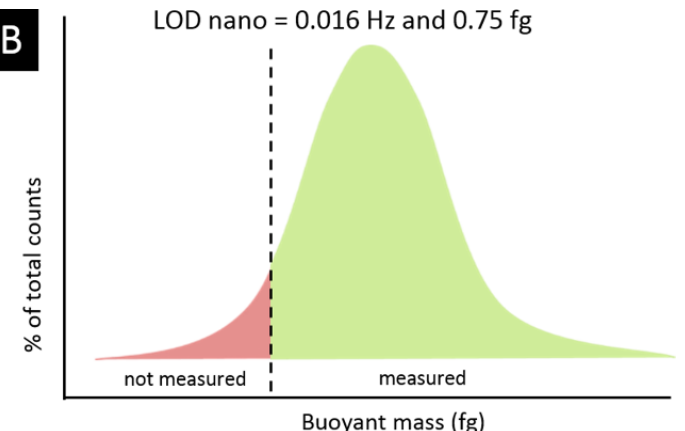

Figure 5. Impact of the LOD on particle measurement: the illustrations exemplify, in an oversimplified manner, why (A) the Lab-GO was poorly measured with the microsensor (high fraction of the flakes below the LOD), while (B) the nanosensor improved the measurement.

of different batches of materials. This tool could be also used to evaluate the mass changes in GBMs upon reduction, functionalisation and adsorption, or to investigate the behaviour of GBMs in different solvents. The main advantages that RMM brings are the possibility to perform measurements directly in the desired solvents (as opposed to microscopy in dry state) and applying non-spherical models (in comparison to DLS or NTA). The limitations of the technique rely on the dependence of detection limit primarily on the density and size of the material to be tested. The graphic in figure 5 illustrates how the LOD impacted on the RMM measurement of our Lab-GO.
The development of suspended resonating sensors with higher sensitivity towards less dense and smaller sheets would be of great benefit for more accurate measurements of the mass and number of graphenebased platelets in dispersions.

Examples where RMM might be useful are nanomedicine and nanosafety, where, similar to the lateral size (Ma et al 2015, Vranic et al 2018), the mass or number of sheets are complementary information for understanding the effects of 2D materials on biological systems. Although most often the effects of GBMs in cell culture and animal models are expressed as a function of mass concentration, we have seen 
here that, when comparing different materials, the same mass can equate to different numbers of sheets. The buoyant mass and particle count could be used to better bridge the gap between the physicochemical properties of 2D materials and their effects on living systems.

\section{Conclusions}

Firstly, this study emphasises the potential of RMM as a novel technique for the characterisation of $2 \mathrm{D}$ materials, providing unique information such as the mass and number of individual particles or aggregates in suspension. RMM offers an alternative for the quantification of 2D materials in dispersion when their optical properties cannot be explored.

Secondly, this paper highlights the importance of thoroughly describing and understanding different types of GBMs defined by similar nomenclature. Here, we compared a lab-prepared GO with two different commercially available GO aqueous dispersions. We report for the first time that the mass and concentration of GO sheets can be measured using suspended resonating sensors. Significant differences in terms of average buoyant mass and particle counts were identified between the three different types of GO, results which were in agreement with particle size and thickness by AFM and TEM.

\section{Experimental}

Materials: an aqueous dispersion of small GO sheets prepared as previously described (Dhifaf et al 2016, Artur Filipe et al 2018) was compared with two commercially available GO dispersions. Samples were labelled as Lab GO, Commercial GO-A and Commercial GO-B.

Raman spectroscopy: Raman spectroscopy was performed on drop-casted GO $\left(100 \mu \mathrm{g} \mathrm{ml}^{-1}\right)$ on glass slides using a calibrated micro-Raman spectrometer (Thermo Scientific, UK), with laser of $633 \mathrm{~nm}$ and $0.4 \mathrm{~mW}$. The objective and pinhole aperture were $50 \times($ NA 0.75$)$ and $50 \mu \mathrm{m}$, respectively. Three measurements of different spots were acquired for each sample. The raw data was processed by fluorescence correction and further normalised by the intensity of the $\mathrm{G}$ band.

UV-Visible (UV-VIS) spectroscopy: UV-VIS absorbance spectra were recorded using a Cary 50 Bio UV/Vis spectrophotometer (Varian Inc., Agilent Technologies, UK). Serial dilutions of GO (2.5$20 \mu \mathrm{g} \mathrm{ml}^{-1}$ ) were prepared and their absorbance at 200-800 nm was recorded. All measurements were performed at room temperature, using MilliQ water (Merck-Millipore, UK) as blank.

Atomic force microscopy (AFM): AFM samples were prepared on $0.01 \%$ poly-L-lysine (PLL, Sigma Aldrich, UK) coated mica substrates (Agar Scientific, UK). PLL was adsorbed on freshly cleaved mica for 1-2 min, then the substrate was gently washed with
MilliQ water and the excess was removed using filter paper (Whatman ${ }^{\mathrm{TM}}$ cat. no. 1001-070, grade no. 1). Next, GO suspension $\left(100 \mu \mathrm{g} \mathrm{ml}^{-1}\right)$ was added and left to adsorb for 1-2 min, followed again by a washing step and removal of the excess. The ared samples were then dried overnight at $37{ }^{\circ} \mathrm{C}$ before imaging. The measurements were done in ScanAsyst mode using ScanAsyst Air probes on a Multimode 8 instrument (Bruker, UK). Data post-processing was done with Nanoscope Analysis version 1.4 (Bruker) dedicated software.

Transmission electron microscopy (TEM): samples for TEM were prepared on 300-mesh carboncoated copper grids $(\mathrm{C} 300 \mathrm{Cu}, \mathrm{EM}$ resolutions, UK). A FEI Tecnai 12 BioTwin microscope (FEI, Eindhoven, NL), operating at $100 \mathrm{kV}$ and equipped with an AMT digital camera (Gatan, UK) was used.

Resonant mass measurements (RMM): RMM was performed on an Archimedes instrument (Malvern Panalytical, UK) operating with Archimedes software version 1.21. Based on the specifications of the instrument, the target concentration for best measurements would be $8 \times 10^{6}$ particles $\mathrm{ml}^{-1}$ for the microsensor and $2 \times 10^{8}$ particles $\mathrm{ml}^{-1}$ for the nano-sensor. The LOD was optimised to $0.04 \mathrm{~Hz}$ for the microsensor and to $0.016 \mathrm{~Hz}$ for the nanosensor. The rationale in setting the LOD was to enable the measurement of lightest possible GO particles without significant background noise. A low background noise would normally be seen as zero to few particle counts in the water reference sample, which was run before each measurement. In addition, the coincidence factor, which is a measurement indicator referring to measurement duplicates (e.g. particle clusters) was monitored to be maintained below $3 \%$, where possible. The particle density was fixed to $2.26 \mathrm{~g} \mathrm{cc}^{-1}$, i.e. the density of crystal graphite (Dismukes et al 1993), and samples were measured in water until 1500 counts were reached. Results are reported as particle mass distribution, particle average mass and counts per mg.

Statistical analysis: the buoyant mass and number counts per milligram were expressed as means with standard deviation $( \pm S D)$ and evaluated for statistical differences by one-way ANOVA using OriginPro SR2 (b9.4.2.380, Academic).

\section{Data availability statement}

All data that support the findings of this study are included within the article (and any supplementary files).

\section{Acknowledgments}

This work was supported through a studentship offered by the EPSRC Centre for Doctoral Training in the Science and Applications of Graphene and Related Nanomaterials (GrapheneNOWNANO CDT; EP/L01548X/1) within the Nanomedicine Lab 
at the Faculty of Biology, Medicine and Health (FBMH) and the National Graphene Institute, The University of Manchester, UK. The microscopy facilities at the FBMH EM Core Facility and the Bioimaging Facility and the Wellcome Trust were accessed in this study. The authors would also like to acknowledge Artur Filipe Rodrigues and Cyrill Bussy for their scientific advice and for critically reading this manuscript. Malvern Panalytical UK funded and offered technical support for all resonance mass measurements.

\section{Authors contribution}

L E C synthesized the GO, characterised all samples, designed the study and wrote the manuscript. T J D and $\mathrm{E}$ A G offered support and advice for Archimedes use and RMM data interpretation. $\mathrm{K} \mathrm{K}$ designed and directed the study, offered intellectual support and contributed to writing the manuscript.

\section{ORCID iDs}

Livia Elena Crica (ㄱ https://orcid.org/0000-00029665-0862

Kostas Kostarelos (1) https://orcid.org/0000-00022224-6672

\section{References}

Alam I, Sa K, Das S, Raiguru J, Subramanyam B, Mahakul P C and Mahanandia P 2018 Preparation of few layer graphene sheets (FLGS) prepared by an electrochemical method IOP Conf. Ser.: Mater. Sci. Eng. 338012063

Artur Filipe R, Leon N, Neus L, Sourav P M, Bengt F, Cyrill B and Kostas K 2018 A blueprint for the synthesis and characterisation of thin graphene oxide with controlled lateral dimensions for biomedicine 2D Mater. 5035020

Bianco A et al 2013 All in the graphene family-A recommended nomenclature for two-dimensional carbon materials Carbon $651-6$

Bianco A 2013 Graphene: safe or toxic? the two faces of the medal Angew. Chem., Int. Ed. 52 4986-97

Bøggild P 2018 The war on fake graphene Nature News and Views (https://doi.org/10.1038/d41586-018-06939-4)

Dhifaf A J, Neus L and Kostas K 2016 Synthesis of few-layered, high-purity graphene oxide sheets from different graphite sources for biology 2D Mater. 3014006

Dimitrios B, Hanene A-B, Hee H B, Dal-Hee M and Kostas K 2013 Prospects and challenges of graphene in biomedical applications Adv. Mater. 25 2258-68

Dismukes J P, Ravi K V, Science E S D, Division T, Division E S H T M and Meeting E S 1993 Proc. Third Int. Symp. on Diamond Materials (Electrochemical Society) (https://books.google.ro/books/about/Proceedings_of_the_ Third_International_S.html?id $\backslash$ protect $\$$ relax $=$ \$vEGZ9loshAsC\&redir_esc $\backslash$ protect\$ $\backslash$ relax $=\$ y$ )

Dreyer D R, Park S, Bielawski C W and Ruoff R S 2010 The chemistry of graphene oxide Chem. Soc. Rev. 39 228-40

Höldrich M, Liu S, Epe M and Lämmerhofer M 2017 Taylor dispersion analysis, resonant mass measurement and bioactivity of pepsin-coated gold nanoparticles Talanta $16767-74$

ISO/TS 80004-13:2017 2017 Nanotechnologies_vocabularypart 13: graphene and related two-dimensional (2D) materials
Kauling A P, Seefeldt A T, Pisoni D P, Pradeep R C, Bentini R, Oliveira R V B, Novoselov K S and Castro Neto A H 2018 The worldwide graphene flake production $A d v$. Mater. 301803784

Kobayashi H, Maeda S, Kashiwa M and Fujita T 2014 Measurement and identification of ultrafine bubbles by resonant mass measurement method 9232 Int. Conf. on Optical Particle Characterization (OPC 2014) (SPIE) p 5

Krueger A B, Hadley J, Cheney P P, Markova N, Carpenter J F and Fradkin A H 2019 Application of a best practice approach using resonant mass measurement for biotherapeutic product characterization J. Pharm. Sci. 108 1675-85

Lewis C L, Craig C C and Senecal A G 2014 Mass and density measurements of live and dead gram-negative and gram-positive bacterial populations Appl. Environ. Microbiol. 80 3622-31

Ma J, Liu R, Wang X, Liu Q, Chen Y, Valle R P, Zuo Y Y, Xia T and Liu S 2015 Crucial role of lateral size for graphene oxide in activating macrophages and stimulating pro-inflammatory responses in cells and animals ACS Nano 9 10498-515

Nejadnik M R and Jiskoot W 2015 Measurement of the average mass of proteins adsorbed to a nanoparticle by using a suspended microchannel resonator J. Pharm. Sci. 104 698-704

Nittayacharn P, Leon A D, Abenojar E C and Exner A A 2018 The effect of lipid solubilization on the performance of doxorubicin-loaded nanobubbles 2018 IEEE Int. Ultrasonics Symp. (IUS) pp 1-4

Novoselov K S, Fal'ko V I, Colombo L, Gellert P R, Schwab M G and Kim K 2012 A roadmap for graphene Nature 490192

Paredes J I, Villar-Rodil S, Martínez-Alonso A and Tascón J M D 2008 Graphene oxide dispersions in organic solvents Langmuir 24 10560-4

Pimenta M A, Dresselhaus G, Dresselhaus M S, Cancado L G, Jorio A and Saito R 2007 Studying disorder in graphite-based systems by Raman spectroscopy Phys. Chem. Chem. Phys. 9 1276-91

Pollard A J 2017 Characterisation of the structure of graphene. Good Practice Guide (United Kingdom: National Physical Laboratory) 145 (available at: https:// eprintspublications.npl.co.uk/id/eprint/8654)

Pollard A J and Clifford C A 2017 Terminology: the first step towards international standardisation of graphene and related 2D materials J. Mater. Sci. 52 13685-8

Pollo M, Mehta A, Torres K, Thorne D, Zimmermann D and Kolhe P 2019 Contribution of intravenous administration components to subvisible and submicron particles present in administered drug product J. Pharm. Sci. 108 2406-14

Randviir E P, Brownson D A C and Banks C E 2014 A decade of graphene research: production, applications and outlook Mater. Today 17 426-32

Son S, Kang J H, Oh S, Kirschner M W, Mitchison T J and Manalis S 2015 Resonant microchannel volume and mass measurements show that suspended cells swell during mitosis J. Cell Biol. 211 757-63

Vranic S, Rodrigues A F, Buggio M, Newman L, White M R H, Spiller D G, Bussy C and Kostarelos K 2018 Live imaging of label-free graphene oxide reveals critical factors causing oxidative-stress-mediated cellular responses ACS Nano 12 1373-89

Weinbuch D, Zolls S, Wiggenhorn M, Friess W, Winter G, Jiskoot W and Hawe A 2013 Micro-flow imaging and resonant mass measurement (Archimedes)-complementary methods to quantitatively differentiate protein particles and silicone oil droplets J. Pharm. Sci. 102 2152-65

Whitby R L D et al 2012 Driving forces of conformational changes in single-layer graphene oxide ACS Nano $63967-73$

Wick P et al 2014 Classification framework for graphene-based materials Angew. Chem., Int. Ed. 53 7714-8 